\title{
Partitioning of Magnetic Helicity in Reconnected Flux Tubes
}

\author{
Andrew N. Wright (iD \\ School of Mathematics and Statistics, University of St. Andrews, Fife KY16 9SS, UK; anw@st-and.ac.uk \\ Received 2019 April 11; revised 2019 May 9; accepted 2019 May 10; published 2019 June 19
}

\begin{abstract}
The reconnection of two flux tubes with footpoints anchored to a plane, such as the photosphere, is considered. We focus on properties of the reconnected flux tubes, specifically their twist, which can be quantified using magnetic helicity. If the tubes are of equal flux $(\Phi)$ and are initially crossed we find the results are dependent upon the relative positioning of their footpoints: (i) nonequipartition of self-helicity is the typical situation; (ii) the total amount of self-helicity in the reconnected tubes lies between 0 and $2 \Phi^{2}$, corresponding to a total twist of between 0 and 2 turns. If the tubes are initially uncrossed the self-helicity of each reconnected tube depends upon footpoint arrangement. However, care needs to be taken when using these results as bringing the tubes together at the reconnection site can introduce twist or writhe, which will also need to be taken into account. In the case where the tubes are side by side and possess some overlap, reconnection may occur without distorting the tubes. For this situation the reconnected tubes will be crossed: (i) equipartition of self-helicity is never met, but can be approached in the limit of the footpoints being quasi-colinear; (ii) the overlying tube always has a self-helicity whose magnitude $>\Phi^{2} / 2$ (it exceeds a half turn); the underling tube's self-helicity magnitude is always $<\Phi^{2} / 2$ (less than a half turn). Our results have a broad application in developing models of reconnecting coronal magnetic fields, as well as in interpreting observations and simulations of these fields.
\end{abstract}

Key words: magnetic fields - magnetic reconnection - methods: analytical

\section{Introduction}

Helicity is a powerful way of quantifying topology in a vector field (Moffatt 1969; Berger \& Field 1984). The original expressions for total helicity within a given volume take the form of volume integrals. However, this can also be viewed in terms of helicities associated with constituent flux tubes (Berger \& Field 1984), which provides a measure of the linkage of different flux tubes and the twist within each tube. Subsequently, this has been taken to the limit of defining field line helicity by Berger (1988), Russell et al. (2015), and Yeates \& Hornig (2016), and references therein.

The fact that magnetic helicity is conserved to a high degree during reconnection (Berger 1984; Russell et al. 2015) has led to its use in laboratory (Taylor 1974), magnetospheric (Song \& Lysak 1989; Wright \& Berger 1989), and solar plasmas (Priest \& Longcope 2017; Threlfall et al. 2018). These studies deduce properties of the post-reconnection state by conserving total helicity.

Studies of magnetic helicity are often given in terms of dense vector calculus integrals. However, there are surprisingly simple and elegant geometrical interpretations to many of these integrals that can facilitate their ready application with little effort. For example, if two linked (and internally untwisted) loops of equal flux undergo reconnection to produce a single loop, that loop will have an internal structure equivalent to a twist of two turns.

In this paper we provide more geometrical properties of magnetic helicity that will aid its application to magnetic fields emerging from a plane. The paper is structured as follows: Section 2 provides the properties of magnetic helicity we require; Sections 3 and 4 consider two tubes with footpoints anchored in a plane, and derive properties of the final states that can be achieved following reconnection-specifically the amount of twist in each tube. Finally, Section 5 summarizes our results and provides some concluding remarks.

\section{Key Helicity Properties}

\subsection{Magnetic Helicity and Gauss Linkage}

A magnetic field $\boldsymbol{B}$ and its vector potential $\boldsymbol{A}$ can be used to define the (ordinary) magnetic helicity as (Moffatt 1969)

$$
\mathcal{H}=\int \boldsymbol{A} \cdot \boldsymbol{B} d V
$$

When $V$ is a simply connected closed volume with no magnetic field passing through its boundary $\mathcal{H}$ is gauge invariant. If the magnetic field exists in the form of closed loops, or flux tubes, there exists a close connection with the Gauss Linking Integral:

$$
\mathcal{L}_{1,2}=\frac{1}{4 \pi} \oint_{\gamma_{1}} \oint_{\gamma_{2}} \frac{\boldsymbol{r}_{1}-\boldsymbol{r}_{2}}{\left|\boldsymbol{r}_{1}-\boldsymbol{r}_{2}\right|^{3}} \cdot\left(d \boldsymbol{r}_{1} \times d \boldsymbol{r}_{2}\right) .
$$

Here $\gamma_{1}$ and $\gamma_{2}$ are two nonintersecting differentiable closed curves that are integrated along using $\boldsymbol{r}_{1}$ and $\boldsymbol{r}_{2}$ respectively. If the curves are linked in a similar fashion to the magnetic flux tubes shown at the top of Figure 1, the Gauss Linking Number is 1 . Geometrically this is found from half the sum of signed crossings. (There are two positive crossings in this example.) For comparison, the magnetic helicity of the flux tubes at the top of Figure 1 (if they have magnetic fluxes $\Phi_{1}$ and $\Phi_{2}$ and no internal structure such as twist) is

$$
\mathcal{H}=N \Phi_{1} \Phi_{2}=2 \mathcal{L}_{1,2} \Phi_{1} \Phi_{2},
$$

and may be thought of as proportional to a flux-weighted Linking Number (Berger \& Field 1984). In Equation (3), $N$ is the number of signed crossings. Evidently, $N=2 \mathcal{L}_{1,2}$ (Berger 1986).

The magnetic helicity $(\mathcal{H})$ also has a topological interpretation for a single flux tube like that at the bottom of Figure 1, and is related to the number of turns the field lines make over one traversal of the tube. The number of turns is signed 


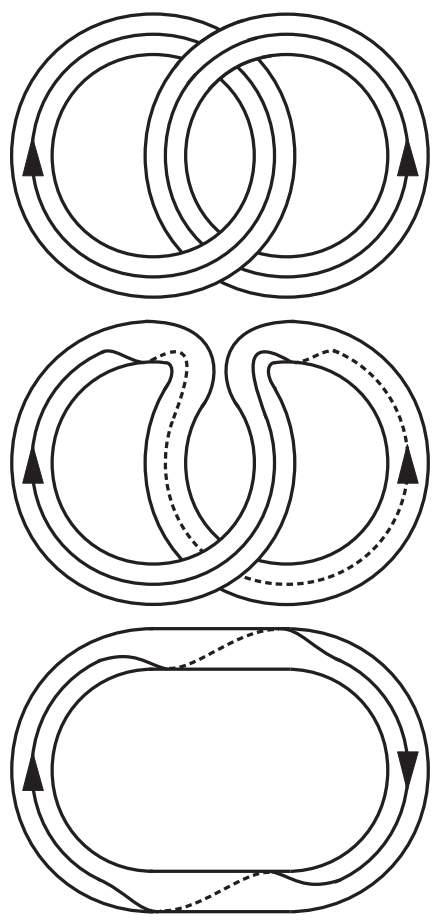

Figure 1. Various flux tube configurations. The top one has two linked flux tubes with no twist or writhe. The middle configuration has twist $(T=1)$ and writhe of the tube axis $(\mathcal{W}=1)$. The bottom tube has no writhe but a twist of two turns $(T=2)$.

positive/negative for right/left handed turns. For $T$ net turns

$$
\mathcal{H}=T \Phi^{2}
$$

for a tube with an axial flux $\Phi$ (Berger $\&$ Field 1984). The tube at the bottom of Figure 1 has $T=2$.

The tube at the center of Figure 1 has a single turn but also writhe (the crossing of the tube axis with itself), which also contributes to the helicity (Berger \& Field 1984). If the tubes are thin and their axes are approximately coplanar, the middle configuration has $T=1$ and a writhe number $\mathcal{W}=1$. Calugareaunu (1959) and White (1969) showed that the sum of $T$ and $\mathcal{W}$ is conserved. Indeed, a careful inspection of middle and bottom tubes shows that one can be deformed onto the other using ideal motions.

\subsection{Self-helicity and Mutual Helicity}

When flux tubes have both linkage with other tubes and internal structure the magnetic field may be broken down into subfields corresponding to each tube and its corresponding vector potential. Suppose we label the tubes with a number such that the first tube has magnetic field $\boldsymbol{B}_{1}$ and corresponding vector potential $\boldsymbol{A}_{1}$. The contribution to $\mathcal{H}$ from $\boldsymbol{A}_{i} \cdot \boldsymbol{B}_{i}$ is a measure of how much the field lines in tube $i$ are linked to themselves, and is denoted by $\mathcal{H}_{i}$. Contributions to $\mathcal{H}$ from $\boldsymbol{A}_{i} \cdot \boldsymbol{B}_{j}$ and $\boldsymbol{A}_{j} \cdot \boldsymbol{B}_{i}$ (denoted by $\mathcal{H}_{i j}$ and $\mathcal{H}_{j i}$ ) measure how much flux tube $\boldsymbol{B}_{i}$ is linked with tube $\boldsymbol{B}_{j}$ and how much flux tube $\boldsymbol{B}_{j}$ is linked with tube $\boldsymbol{B}_{i}$. Evidently these must be the same: $\mathcal{H}_{i j}=\mathcal{H}_{j i}$. The total helicity is (Berger \& Field 1984)

$$
\mathcal{H}=\sum_{i} \mathcal{H}_{i}+\sum_{i, j(i \neq j)} \mathcal{H}_{i j}
$$

There is a loose similarity with the interpretation of inductance of electrical circuits that measures the linkage of a circuit with its own magnetic flux (self-inductance) or with the magnetic flux generated by other circuits (mutual inductance). This led Wright \& Berger (1989) to introduce the terms "selfhelicity" and "mutual helicity" for the two contributions $\mathcal{H}_{i}$ and $\mathcal{H}_{i j}$. Note that the mathematical form of the integrals for helicities and inductances are not identical (Demoulin et al. 2006): inductance measures the linkage of the magnetic field produced by a circuit with another (possibly the same) circuit, whereas helicity would correspond to the linkage of one circuit with another (possibly the same) circuit.

The tubes at the top of Figure 1 are internally untwisted and have no writhe, so $\mathcal{H}_{i}=0$ for both tubes. The helicity $\mathcal{H}=2 \Phi_{1} \Phi_{2}$ arises solely from mutual helicity. In contrast, the single tube at the bottom of the figure has no contribution from mutual helicity (as there are no other tubes to link with), and $\mathcal{H}=\mathcal{H}_{i}=2 \Phi^{2}$ arises solely from the internal twist of the field within the tube. Self-helicity not only arises from the twisting of field lines within a tube, but can also be associated with the writhe of the tube axis. Both the middle and bottom tubes in Figure 1 have the same self-helicity, but in the middle one there are contributions associated with the single turn and the writhe of the tube axis. Self-helicity $\left((T+\mathcal{W}) \Phi^{2}\right)$ is associated with the Calugareanu Invariant (Berger \& Field 1984; Moffatt \& Ricca 1995).

\subsection{Relative Helicity}

If the magnetic field passes through the surface bounding the volume under consideration, $\mathcal{H}$ is not gauge invariant and so no longer has a topological interpretation. This led Berger \& Field (1984) to develop "relative helicity." Besides the formulation given by Berger \& Field (1984) there are alternative, but equivalent, expressions provided by Jensen \& Chu (1984), Finn \& Antonsen (1985) and Berger (1988). The relative helicity $(H)$ is gauge invariant and has several elegant topological interpretations. The ideas of self- and mutual helicities also remain valid.

Consider the space between to infinite parallel planes, such as that in Figure 2. If flux tube $i$ contains a uniformly twisted field with a signed number of turns $T$ on passing between the planes, then it has a relative self-helicity of

$$
H_{i}=T \Phi_{i}^{2}
$$

If the footpoints of the two tubes $(i$ and $j$ ) in Figure 2 turn through a signed angle $\phi$ on mapping from one plane to the other, then the contribution to the relative mutual helicity is $H_{i j}+H_{j i}$, where (Berger 1986)

$$
H_{i j}=H_{j i}=\frac{\phi}{2 \pi} \Phi_{i} \Phi_{j}
$$

If two flux tubes have both of their footpoints on a single plane (as in Figure 3) the self-helicity result (6) still applies, but the mutual helicity terms are defined as (Berger 1986)

$$
\begin{aligned}
& H_{i j}=H_{j i}=\frac{\rho_{1}+\nu_{1}}{2 \pi} \Phi_{i} \Phi_{j} . \\
& H_{i j}=H_{j i}=\frac{\rho_{2}-\nu_{2}}{2 \pi} \Phi_{i} \Phi_{j} .
\end{aligned}
$$

These formulae can be derived by integrating the appropriate $d H_{i j} / d t$ equation in, for example, Berger (1988), Wright \& Berger (1990), and Pariat et al. (2005). 

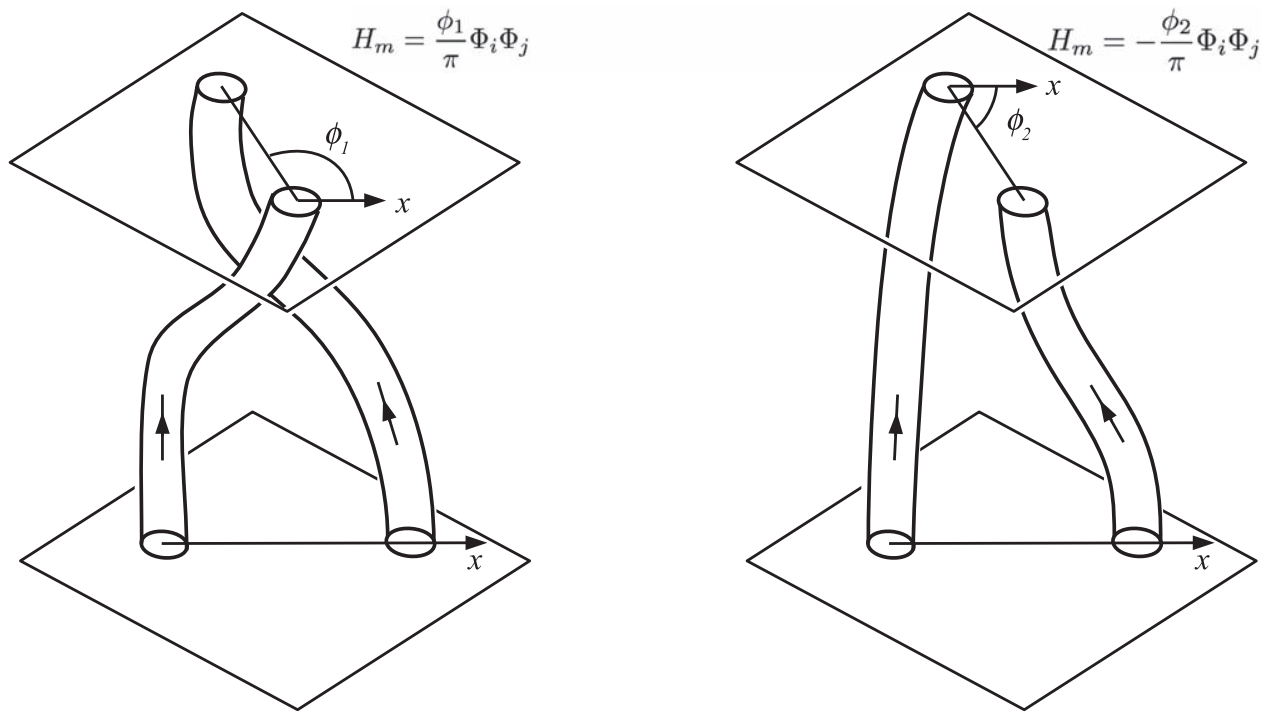

Figure 2. Two flux tubes (with fluxes $\Phi_{i}$ and $\Phi_{j}$ ) extend between two parallel planes. The relative mutual helicity of the tubes is determined by the angle the line joining the footpoints turns through when moving from the lower to the upper plane. If the fluxes are equal $\left(\Phi_{i}=\Phi_{j}=\Phi\right)$ and the tubes in the first configuration reconnect to give the second, without footpoint motion, the angle at which the tubes turn around each other changes by exactly $-\pi$, and the relative mutual helicity changes by $-\Phi^{2}$. (This is strictly true if the tube width is much less than the footpoint separation.)

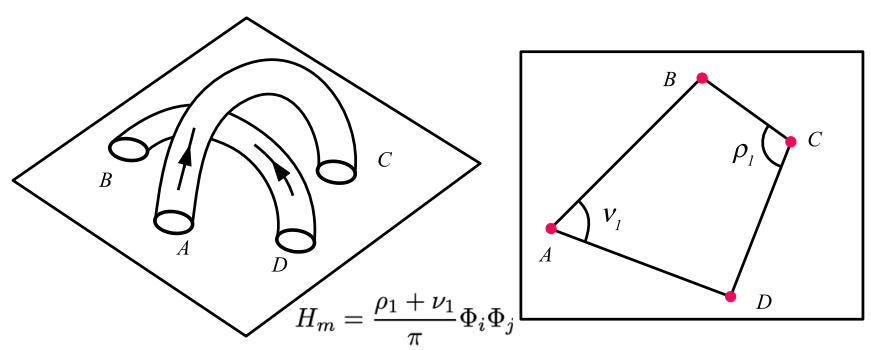

(a)

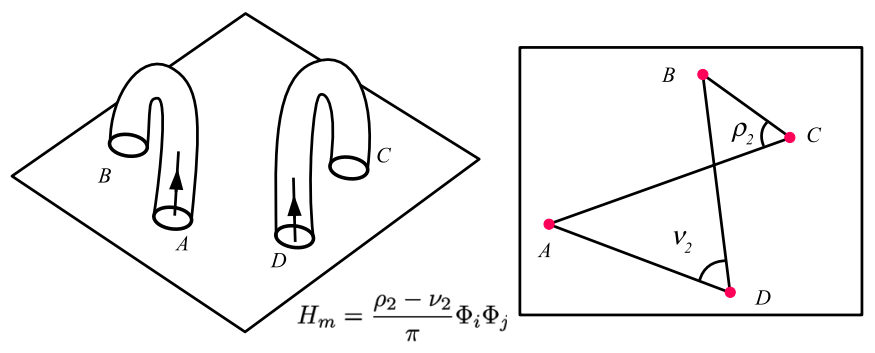

(b)

Figure 3. Two flux tubes (with fluxes $\Phi_{i}$ and $\Phi_{j}$ ) have footpoints fixed in a common plane. The relative mutual helicity of the tubes may be interpreted in terms of the angle subtended by one tube about the other tube's footpoints, as indicated for crossed and uncrossed arrangements.

Finally, we note that the direction of the magnetic fields is important. For example, if the direction of $\boldsymbol{B}_{i}$ is reversed, the sign of $\Phi_{i}$ will change as will those of $H_{i j}$ and $H_{j i}$.

\subsection{Helicity Conservation}

During reconnection the topology and linking of flux elements can change dramatically. Although there is some change of helicity during reconnection (the rate of change of helicity density is $-2 \eta \boldsymbol{j} \cdot \boldsymbol{B}$, where $\eta$ is the resistivity), it is relatively small compared to energy dissipation, and in the solar corona (Berger 1984) and the magnetosphere (Wright \& Berger 1989) it is a good approximation to treat helicity as being conserved. Near-conservation has also been established within the field line helicity formulation (Russell et al. 2015). Wright (1999) noted a geometrical interpretation to the change in helicity during reconnection; it is associated with the change in linkage of flux embedded in the diffusion region where reconnection occurs. Although it is common to talk about helicity dissipation, Wright shows how the helicity can increase during reconnection, so it is more correct to say that helicity nonconservation (rather than dissipation) is small during reconnection.

Helicity conservation is a powerful tool for constraining properties of the field following reconnection. It was first used by Taylor (1974) to determine the lowest energy equilibrium when helicity is conserved in a Reverse Field Pinch. Subsequently, a similar approach has been applied to coronal fields (Hood et al. 2009). It can also be used to determine the amount of twist in dynamic reconnected flux tubes when they are far from equilibrium, and was first applied by Wright \& Berger (1989) in a magnetospheric context.

The power of helicity conservation can be appreciated by considering the situation where the flux tubes in Figure 1 all have the same axial flux $\Phi$ : the three configurations all have $\mathcal{H}=2 \Phi^{2}$. In the top one this comes from mutual helicity (selfhelicity is zero). Following reconnection at the upper crossing, the middle configuration may be achieved, which only has a self-helicity. (The mutual helicity is zero as there is now only a single tube.) The bottom loop can be arrived at by ideal motions of the middle tube, and so must have a helicity due to internal twisting, $\mathcal{H}=T \Phi^{2}$. Thus helicity conservation can be used to deduce that the final configuration has exactly two complete turns of twist.

Wright \& Berger (1989) used properties of the tubes in Figure 2 to show that if the tubes (of flux $\Phi$ ) started with selfhelicities of zero in the first configuration, then reconnected to the second state (with no footpoint motion), then each reconnected tube has a final self-helicity equivalent to exactly a half twist $(T=1 / 2)$. 
Given that the tubes in Figure 3(a) (if both have flux $\Phi$ ) can reconnect to produce the configuration in Figure 3(b), it is natural to ask if the twist generated in the reconnected tubes has a simple, exact value as in the examples above. Indeed, Priest \& Longcope (2017) have considered reconnecting the uncrossed tubes in Figure 3(b) to give the crossed configuration in Figure 3(a), and have shown this situation is not so simple. They found that the total amount of self-helicity in the final configuration depended upon the footpoint arrangement, in contrast to the result corresponding to Figure 2. Moreover, the way self-helicity is partitioned between the reconnected flux tubes is known for Figure 2 (Wright \& Berger 1989 prove there is equipartition of self-helicity). For configurations similar to those in Figure 3, Priest et al. (2016) introduced the "helicity equipartition conjecture" to allow the exploitation of helicity conservation in modeling coronal fields.

The remainder of this paper is devoted to evaluating the selfhelicities of reconnected tubes when changing between the configurations in Figure 3 for arbitrary footpoint positions. Hence we are concerned exclusively with relative helicity, which, for brevity, we refer to simply as helicity hereafter.

\section{Crossed Tubes}

This section focuses on reconnecting the crossed configuration in Figure 3(a) to produce the uncrossed configuration in Figure 3(b). We consider general footpoint placements and identify a general criteria for when the equipartition conjecture holds.

\subsection{General Footpoint Arrangement}

To aid visualization of twist we shall take the flux tubes to have the form of thin ribbons, or flux sheets. The initial configuration of Figure 3(a) is represented as flux sheets in Figure 4(a) where the sheets have relaxed onto the footpoint plane for a simple depiction of the system, and is viewed looking down on that plane. The positioning of footpoints is quite general, with the proviso that the tubes cross one another. We can introduce imaginary vertical parallel planes (indicated by the blue lines) to provide a subdomain that enables the determination of twist added to the sections of tube in this region.

A close-up view of the subdomain between the two blue planes in Figure 4(b) is shown on the left of Figure 5(a) prior to reconnection. The middle of Figure 5(a) shows the sheets immediately after reconnection. A careful consideration of the order of reconnection of field lines in the flux sheets allows an easy visualization of twist and provided enough insight to allow Wright (1987) to argue that the reconnected tubes would have a half turn each, suggesting the equipartion conjecture can be applied here. Ideal motions can then allow the flux sheets to adopt the configuration at the right of Figure 5(a). This configuration is topologically identical to the situation in Figure 2: the tubes initially have $\phi_{1}=\pi$ and no self-helicity, so Equations (6) and (7) give a total $H=\Phi^{2}$.

The reconnected tubes (middle configuration of Figure 5(a)) do not twine about each other $\left(\phi_{2}=0\right)$ so $H_{i j}=0$, and the conserved helicity $\left(H=\Phi^{2}\right)$ arises from the self-helicities of the two reconnected tubes. In Appendix B of Wright \& Berger (1989) a detailed analysis shows that if reconnection completely unlinks the tubes, then helicity equipartition holds and they will both have a self-helicity of $\Phi^{2} / 2$, which corresponds to a half turn of twist. Of course, it is possible for subsequent reconnection within a given tube to produce more complex field line braiding within the tube, but the selfhelicity will remain unchanged. (A half turn is the simplest internal structure for a self-helicity of $\Phi^{2} / 2$.) In the right-hand configuration of Figure 5(a) the tubes have relaxed following reconnection, and the half turn is evident.

Care must be taken when applying ideal motions to bring flux tubes together to reconnect that any writhe or twist introduced is accounted for, as is done in the configuration on the left of Figure 5(b). In this case the analysis used for Figure 5(a) can be applied to a localized region around the reconnection site to deduce that a half twist is added to each tube here, which can then be added to any twist elsewhere on the tube. The right-hand configuration shows how one tube has a complete turn, while the other is untwisted.

The importance of reconnection occurring along a line in the middle configuration of Figure 5(a) that results in complete separation of the reconnected tubes on the right should be stressed. For example, it is possible to form reconnected tubes going from $A$ to $B$ and $D$ to $C$, but some of the field lines of one tube may still link with the other. At the heart of this issue is the requirement to have reconnection along a line that coincides with the crossings of the left, middle, and right lines of $A C$ with the right, middle, and left lines of $B D$, respectively. Reconnection at other crossings is also allowed, and will redistribute the self-helicity as interior structure of the reconnected tubes that is not a simple half twist (see Wright \& Berger 1989 for a more detailed account.)

The issue described above regarding details of reconnection can be circumvented by adjusting the orientation of the ribbons we start with in Figure 4. Suppose the ribbons in Figure 4(a) are chosen to lie in a vertical plane, so that they would be viewed edge-on. The corresponding close-up of Figure 4(b) is shown in Figure 5(c). Notice how the ribbons meet edge-on and will now reconnect at a point in the order right, middle, and left of $A C$ with left, middle, and right of $B D$. The same helicity analysis applies as that used in Figure 5(a), and the reconnected tubes both have a twist $T=+1 / 2$.

Reconnection at a line, rather than a point, is probably more likely to occur in nature. Indeed the sheet of flux in Figure 5(a) could be thought of as part of a 3D flux tube and corresponds to the flux reconnected over a short time interval (Wright 1987). However, the edge-on ribbons in Figure 5(c) reconnecting at a point provide a clean unambiguous final state that may be preferred in some thought experiments.

Returning to the reconnected tubes in Figure 4(b), it is tempting to let the tubes relax (c) and claim they both have a half turn of twist. This turns out not to be the case and can be appreciated by considering how the footpoints would need to be turned in order to untwist the tubes, as in (d). We introduce the angles $\theta_{A}, \theta_{B}, \theta_{C}$, and $\theta_{D}$ in $(\mathrm{g})$ to facilitate this. These angles have values between 0 and $\pi$.

Rotating a tube about the center of its footpoint was shown by Wright \& Berger (1990) to cause a flux of self-helicity into the tube across the boundary (see also Berger 1988, Pariat et al. 2005, and Demoulin et al. 2006). Integrating their equation for $d H_{i} / d t$ in time gives the change in $H_{i}$. We can concisely summarize this for our purposes here: rotating a flux tube about its axis through a signed angle $\Delta \theta$ about the (upward) normal 


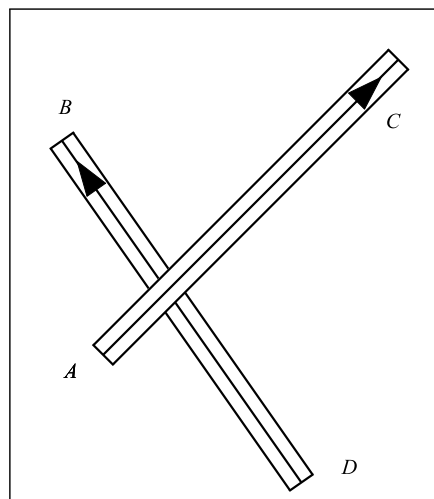

(a)
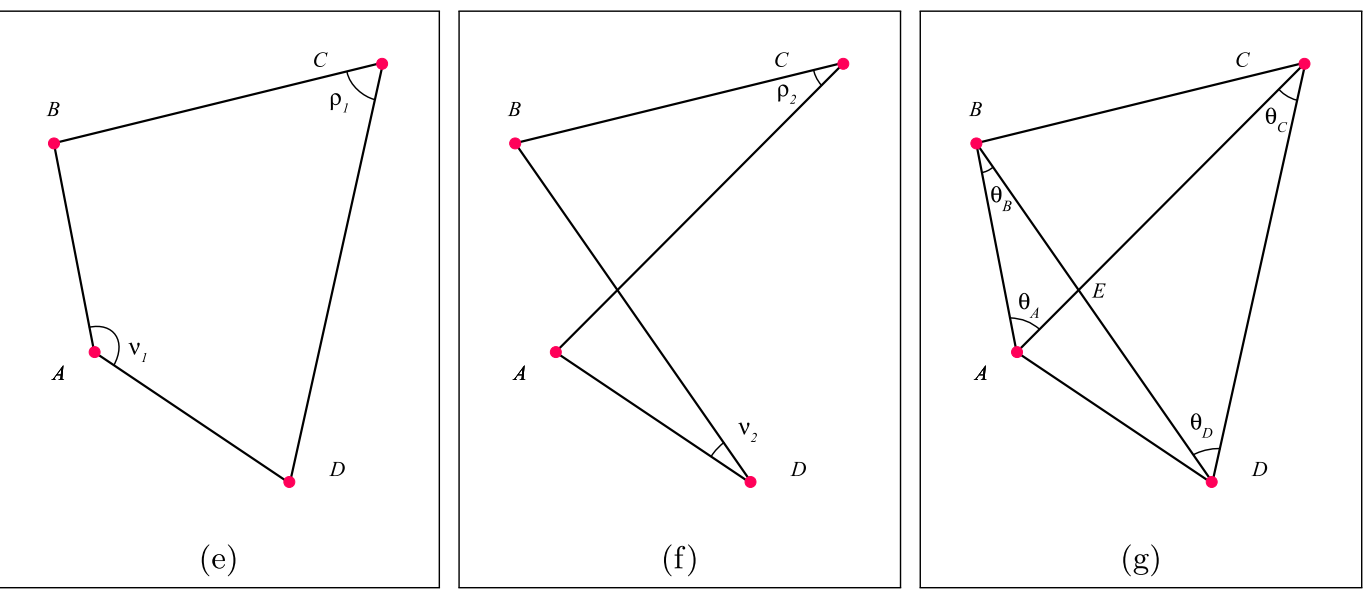

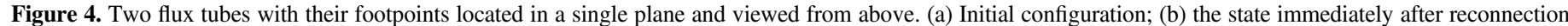

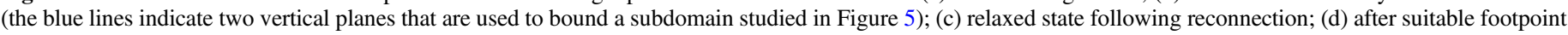

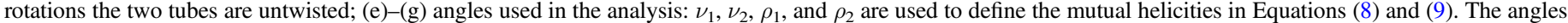
$\theta_{A}, \theta_{B}, \theta_{C}$, and $\theta_{D}$ are used to identify the angles the corresponding footpoints in (c) need to be turned through to produce the untwisted ribbons in (d).

direction of the plane adds a self-helicity of

$$
\Delta H_{i}=-\frac{\Delta \theta}{2 \pi} \Phi^{2} .
$$

To untwist tube $A B$ in Figure 4(c) we rotate about footpoint $A$ by an angle $\theta_{A}$ (adding $\Delta H_{i}=-\left(\theta_{A} / 2 \pi\right) \Phi^{2}$ ) and rotate about $B$ by $\pi-\theta_{B}$ (adding $\left.\left.\Delta H_{i}=-\left(\left(\pi-\theta_{B}\right) / 2 \pi\right) \Phi^{2}\right)\right)$. If the tube $A B$ in Figure 4(d) is now untwisted (and has a self-helicity of zero) it follows that before the rotations were applied to (c) it had a self-helicity of

$$
H_{A B}=\frac{\pi+\theta_{A}-\theta_{B}}{2 \pi} \Phi^{2} .
$$

In a similar fashion tube $C D$ in Figure 4(c) can be untwisted by rotating about $D$ by $-\theta_{D}$ (adding $\Delta H_{i}=\left(\theta_{D} / 2 \pi\right) \Phi^{2}$ ) and rotating about $C$ by $\theta_{C}+\pi$ (adding $\left.\left.\Delta H_{i}=-\left(\left(\theta_{C}+\pi\right) / 2 \pi\right) \Phi^{2}\right)\right)$ to arrive at the untwisted state in (d). This suggests the self-helicity of tube $C D$ in (c) is

$$
H_{C D}=\frac{\pi+\theta_{C}-\theta_{D}}{2 \pi} \Phi^{2} .
$$

If this interpretation is correct we should find the total helicity of the untwisted tubes in Figure 4(a) (due to the mutual helicity (8)) should equal the total helicity of the twisted reconnected tubes in (c), which arises from mutual helicity (9) and the proposed self-helicities $H_{A B}$ and $H_{C D}$,

$$
\begin{aligned}
\frac{\rho_{1}+\nu_{1}}{\pi} \Phi^{2}= & \frac{\rho_{2}-\nu_{2}}{\pi} \Phi^{2}+\frac{\pi+\theta_{A}-\theta_{B}}{2 \pi} \Phi^{2} \\
& +\frac{\pi+\theta_{C}-\theta_{D}}{2 \pi} \Phi^{2} .
\end{aligned}
$$

The validity of this equation can be established by noting that not all of the angles are independent. Evidently in Figure $4(\mathrm{~g}) \angle A E B=\angle C E D$ and $\angle B E C=\angle A E D$, which provide the identities

$$
\begin{gathered}
\theta_{A}+\theta_{B}=\theta_{C}+\theta_{D} \\
\nu_{1}+\nu_{2}+\rho_{1}-\rho_{2}+\theta_{D}-\theta_{A}=\pi .
\end{gathered}
$$

Substituting these identities into (13) confirms, after a little algebra, that helicity is indeed conserved and suggests the proposed forms for $H_{A B}$ and $H_{C D}$ are correct.

\subsection{General Equipartition Criterion}

We are now in a position to deduce when equipartition of helicity will occur: we require $H_{A B}=H_{C D}$, which (after eliminating $\theta_{D}$ using (14)) becomes

$$
\theta_{A}=\theta_{C}, \quad \text { (equivalent to } \theta_{B}=\theta_{D} \text { ). }
$$

(The equivalence comes from the identity (14).) 

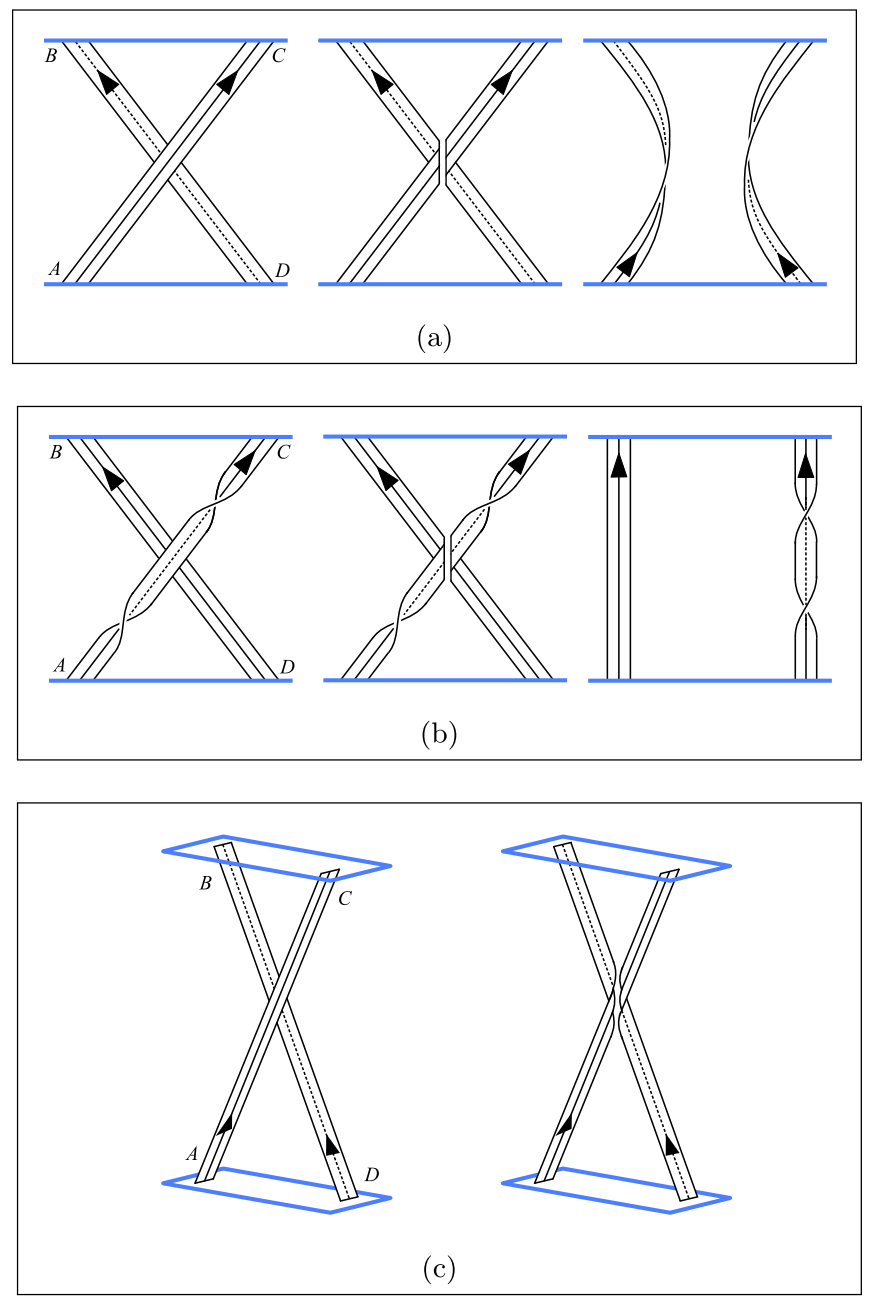

Figure 5. Close-up view of the region between the two vertical planes shown in Figure 4(b). Panels (a) and (b) show the initial state (left), the state immediately after reconnection (center), and the relaxed state (right). Panel (b) shows the importance of accounting for twist or writhe away from the reconnection site when determining the final configuration. Panel (c) shows the situation if an alternative ribbon orientation is adopted.

The geometric interpretation of $\theta_{A}=\theta_{C}$ in Figure $4(\mathrm{~g})$ is clear: the footpoints of the two reconnected tubes lie along the lines $A B$ and $C D$, and we require these lines to be parallel. Indeed any footpoint arrangement for which $A B$ and $C D$ are the bases (parallel sides) of a trapezium will exhibit equipartition, even if highly obtuse (sheared).

\subsection{Degree of Nonequipartition}

When the footpoints do not form a trapezium there will be an imbalance in the self-helicity of each reconnected flux tube. We can quantify the relative imbalance of this partitioning by defining (and using (11), (12), and (14))

$$
\chi=\frac{H_{A B}-H_{C D}}{H_{A B}+H_{C D}} \equiv \frac{\theta_{A}-\theta_{C}}{\pi-\theta_{B}+\theta_{C}} .
$$

As expected, $\chi=0$ if $\theta_{A}=\theta_{C}$ and we recover equipartition. It is important to note that although the angles $\theta_{A}, \theta_{B}, \theta_{C}$, and $\theta_{D}$ are all between 0 and $\pi$, they are not completely arbitrary: to use the equations in this section we need to have an initial configuration in which the tubes are crossed (Figure 3(a)), so $\theta_{A}$ cannot exceed $\pi-\theta_{B}$ (Figure $4(\mathrm{~g})$ ).
It is possible to identify a few key cases to illustrate the extent to which nonequipartition can occur. Figures 6(a)-(c) show an extreme case with $\theta_{A} \rightarrow \pi, \theta_{B} \rightarrow 0, \theta_{C} \rightarrow 0$, and $\theta_{D} \rightarrow \pi$. (For clarity, these cases are sketched a little off the limiting values.) From (11), (12), and (17) we expect $H_{A B}=\Phi^{2}, H_{C D}=0$, and $\chi=+1$. The state immediately after reconnection is shown in (b) and the relaxed state in (c), where the uniform twist equivalent to the self-helicity is also indicated. It is remarkable that equipartition is totally violated, and all the self-helicity goes into just tube $A B$.

By repositioning the footpoints it is possible to get all of the self-helicity deposited in tube $C D$. Figures $6(\mathrm{~d})-(\mathrm{f})$ illustrate this case $\left(\theta_{A} \rightarrow 0, \theta_{B} \rightarrow \pi, \theta_{C} \rightarrow \pi\right.$, and $\left.\theta_{D} \rightarrow 0\right)$, and the analysis suggests $H_{A B}=0, H_{C D}=\Phi^{2}$ with an imbalance of $\chi=-1$, equivalent to $A B$ being untwisted and $C D$ having one full turn.

\subsection{Bounds on Self-helicity}

In addition to the degree of nonequipartition, it is of interest to calculate the amount of total self-helicity that reconnecting crossed tubes can liberate. The minimum that can be released is 0 , and arises when both the initial and final mutual helicities are zero $\left(\nu_{1} \rightarrow 0, \quad \rho_{1} \rightarrow 0, \quad \nu_{2} \rightarrow 0\right.$, and $\rho_{2} \rightarrow 0$, which is equivalent to $\theta_{A} \rightarrow 0, \theta_{B} \rightarrow \pi, \theta_{C} \rightarrow 0$, and $\theta_{D} \rightarrow \pi$ ), and is shown in Figures 6(g)-(i). As expected, from (16) we have equipartition of self-helicity, and in this case both reconnected tubes have zero self-helicity-see (11) and (12).

The maximum self-helicity that can be liberated occurs when the initial mutual helicity is maximum $\left(\nu_{1} \rightarrow \pi, \rho_{1} \rightarrow \pi\right)$ having a value of $2 \Phi^{2}$-equivalent to a full link as in the top configuration of Figure 1-and the final mutual helicity is zero $\left(\nu_{2} \rightarrow 0, \rho_{2} \rightarrow 0\right)$. This configuration can be achieved by letting $\theta_{A} \rightarrow \pi, \theta_{B} \rightarrow 0, \theta_{C} \rightarrow \pi$, and $\theta_{D} \rightarrow 0$, and is shown in Figures 6(j)-(1), and both the reconnected tubes have a selfhelicity $\Phi^{2}$ (or 1 full turn). This case obeys equipartition, as expected since $\theta_{A}=\theta_{C}$.

\section{Uncrossed Tubes}

It seems plausible for the tubes in Figure 3(a) to reconnect and produce the configuration in Figure 3(b) as they can naturally come into contact with each other. It is not so obvious that we can start with the uncrossed arrangement in Figure 3(b) and reconnect this to produce the crossed tubes in Figure 3(a). However, this process has been employed by Priest \& Longcope (2017) and shown to occur in simulations by Threlfall et al. (2018), so we present an analysis for this case here. It must be stressed that if the initial uncrossed tubes do not overlap and present a natural reconnection site, then the tubes will need to be distorted before reconnection can occur. Distortions are quite likely to produce internal structure in the form of twisting (e.g., as in Figure 5(b)) or writhe, which would need to be allowed for on top of the self-helicity change associated with reconnection. We focus on three cases where there is some natural degree of overlap and distortion is not required to allow reconnection.

\subsection{BD Overlap}

Figure 7(a) shows footpoints $B$ and $D$ overlapping with the other tube and generating a natural reconnection site. Following the approach of Section 3.1 a careful consideration of the reconnected tubes shows they can be untwisted by the 

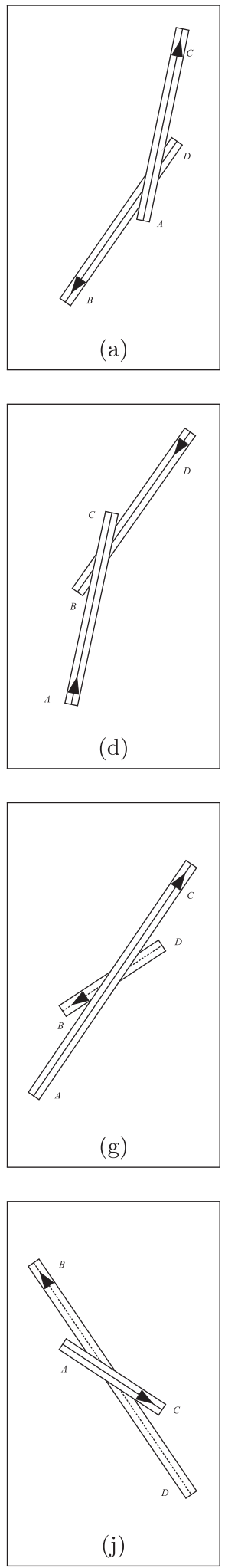
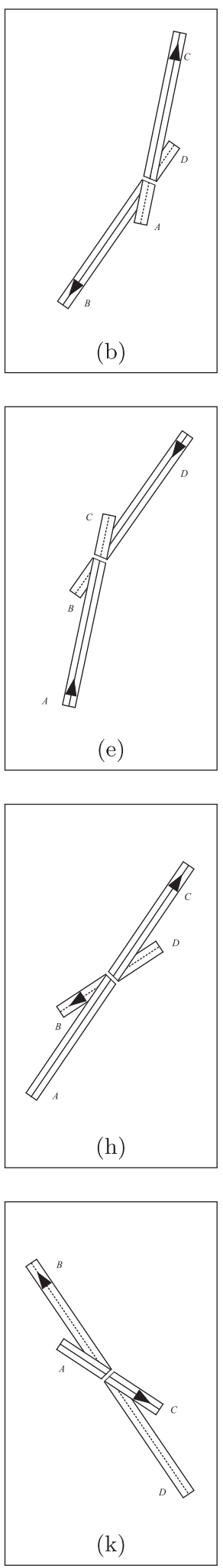
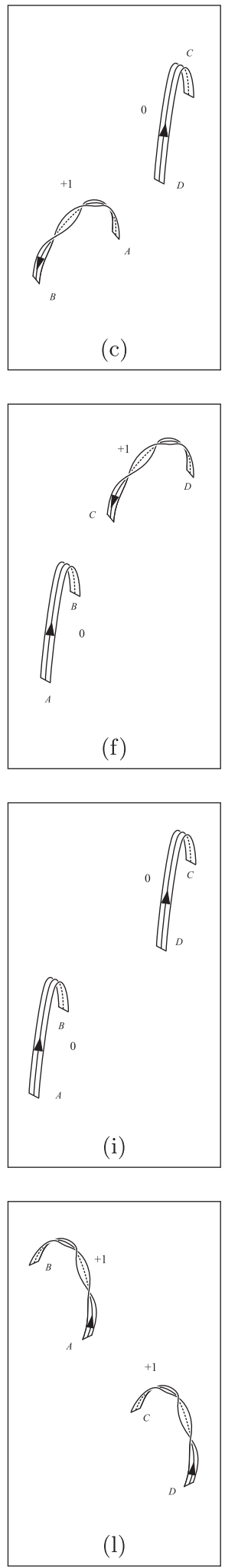

Figure 6. Four examples demonstrating of flux tube reconnection exhibiting extreme behavior. In all examples we have the state prior to reconnection (left), immediately after reconnection (center), and the relaxed state (right). Cases of extreme nonequipartition (a)-(c) and (d)-(f) ; minimum twist produced (g)-(i); maximum total twist produced (j)-(l). In the right-hand figures the signed uniform twist equivalent to the self-helicity is indicated.

following footpoint motions: for $A C$ we rotate about $A$ by $-\left(\theta_{A}+\pi / 2\right)$, adding $\Delta H_{i}=\left(\theta_{A}+\pi / 2\right) \Phi^{2} / 2 \pi$, and rotate about $C$ by $-\left(\theta_{C}+\pi / 2\right)$, adding $\Delta H_{i}=\left(\theta_{C}+\pi / 2\right) \Phi^{2} / 2 \pi$. If the tubes have no self-helicity initially, it follows that after reconnection tube $A C$ has a self-helicity of

$$
H_{A C}=-\frac{\theta_{A}+\theta_{C}+\pi}{2 \pi} \Phi^{2} .
$$



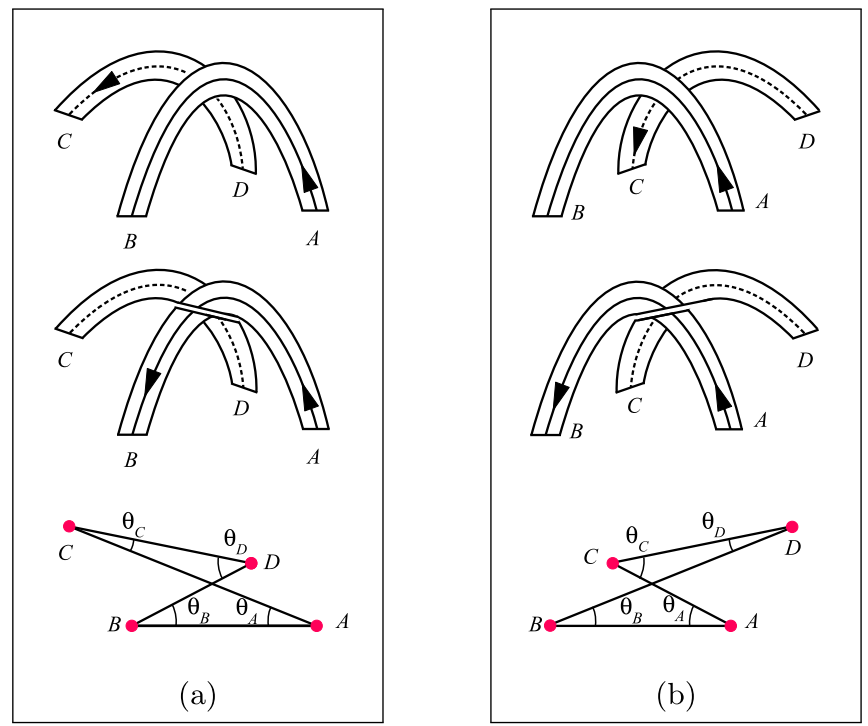

Figure 7. (a) The initial state of uncrossed tubes when footpoints $B$ and $D$ overlap with the other tube (top). Following reconnection, tube $A C$ overlies tube $B D$ (middle). A plan view of the footpoints and relevant angles is also shown (bottom). (b) The initial state of uncrossed tubes when footpoints $A$ and $C$ overlap with the other tube (top). Following reconnection, tube $B D$ overlies tube $A C$ (middle). A plan view of the footpoints and relevant angles is also shown (bottom).

Similarly tube $B D$ is untwisted by rotating about $B$ by $\theta_{B}-\pi / 2$ (adding $\left.\Delta H_{i}=-\left(\theta_{B}-\pi / 2\right) \Phi^{2} / 2 \pi\right)$ and rotating about $D$ by $\theta_{D}-\pi / 2$ (adding $\left.\Delta H_{i}=-\left(\theta_{D}-\pi / 2\right) \Phi^{2} / 2 \pi\right)$, indicating

$$
H_{B D}=\frac{\theta_{B}+\theta_{D}-\pi}{2 \pi} \Phi^{2}
$$

Given the initial and final mutual helicities (from Equations (9) and (8), respectively) it is easy to confirm that the self-helicities above conserve helicity on recalling the identities (14) and (15). Interestingly, $H_{A C}<-\Phi^{2} / 2$ and $H_{B D}>-\Phi^{2} / 2$ (since $\theta_{A}, \theta_{B}, \theta_{C}$, and $\theta_{D}>0$ ) so equipartition is never achieved, although it may be approached in the limit $\theta_{A}$, $\theta_{B}, \theta_{C}$, and $\theta_{D} \rightarrow 0$ and corresponds to both tubes having a selfhelicity of $-\Phi^{2} / 2$ (or -ve half turn). This limit, where the footpoints are almost colinear, is shown in Figure 8(a) viewed side-on before (top) and after (bottom) reconnection. The uniform twist equivalent to the self-helicity is also indicated.

\subsection{AC Overlap}

Figure 7(b) shows footpoints $A$ and $C$ overlapping and generating a natural reconnection site. Again a careful consideration of the reconnected tubes shows they can be untwisted by the following footpoint motions: for $A C$ we rotate about $A$ by $\pi / 2-\theta_{A}$, adding $\Delta H_{i}=-\left(\pi / 2-\theta_{A}\right) \Phi^{2} / 2 \pi$, and rotate about $C$ by $\pi / 2-\theta_{C}$, adding $\Delta H_{i}=-\left(\pi / 2-\theta_{C}\right) \Phi^{2} / 2 \pi$. If the tubes have no self-helicity initially, it follows that after reconnection tube $A C$ has a selfhelicity of

$$
H_{A C}=\frac{\pi-\theta_{A}-\theta_{C}}{2 \pi} \Phi^{2}
$$

Similarly tube $B D$ is untwisted by rotating about $B$ by $\theta_{B}+\pi / 2$ (adding $\left.\Delta H_{i}=-\left(\theta_{B}+\pi / 2\right) \Phi^{2} / 2 \pi\right)$ and rotating about $D$ by $\theta_{D}+\pi / 2$ (adding $\Delta H_{i}=-\left(\theta_{D}+\pi / 2\right) \Phi^{2} / 2 \pi$ ), indicating

$$
H_{B D}=\frac{\theta_{B}+\theta_{D}+\pi}{2 \pi} \Phi^{2}
$$

Again, the self-helicities above and the appropriate mutual helicities can be shown to conserve total helicity. Since $H_{A C}<\Phi^{2} / 2$ and $H_{B D}>\Phi^{2} / 2$, it follows that equipartition never occurs for this case, although it may be approached in the limit $\theta_{A}, \theta_{B}, \theta_{C}$, and $\theta_{D} \rightarrow 0$ and corresponds to both tubes having a self-helicity of $+\Phi^{2} / 2$ (or + ve half turn). This limit, where the footpoints are approximately colinear is shown sideon in Figure 8(b) both before (top) and after (bottom) reconnection.

\subsection{Quasi-colinear Footpoints}

Considering Figure 7(a) it is evident that the uncrossed tubes will present most naturally for reconnection if they are side-byside and have some overlap. This will result if $\theta_{A} \ll 1$ and $\theta_{C} \ll 1$ and we require $0<\theta_{B}<\pi / 2$ and $0<\theta_{D}<\pi / 2$ to preserve the overlap. We refer to this type of highly aligned footpoint arrangement as quasi-colinear. Under these assumptions, the self-helicities of the reconnected tubes reduce to

$$
\tilde{H}_{A C} \approx-\frac{1}{2} \Phi^{2}, \quad \tilde{H}_{B D}=\frac{\theta_{B}+\theta_{D}-\pi}{2 \pi} \Phi^{2}
$$

so the overlying tube $(A C)$ always has close to a -ve half turn, but the underlying tube $(B D)$ has $-\Phi^{2} / 2<\tilde{H}_{B D}<0$ so can range from being untwisted to having a -ve half turn.

A similar analysis for Figure 7(b) indicates the quasi-colinear arrangement results if $\theta_{B} \ll 1$ and $\theta_{D} \ll 1$ and we require $0<\theta_{A}<\pi / 2$ and $0<\theta_{C}<\pi / 2$ to preserve the overlap. Under these assumptions, the self-helicities of the reconnected tubes reduce to

$$
\tilde{H}_{A C}=\frac{\pi-\theta_{A}-\theta_{C}}{2 \pi} \Phi^{2}, \quad \tilde{H}_{B D} \approx \frac{1}{2} \Phi^{2} .
$$

Now the overlying tube $(B D)$ always has close to a +ve half turn, but the underlying tube $(A C)$ has self-helicity corresponding to a state somewhere between untwisted to having a +ve half turn.

There is one other quasi-colinear case to consider and this is shown in Figure 8(c). The top configuration shows both footpoints $C$ and $D$ overlapping with tube $A B$. There is no natural reconnection site here, but allowing $C D$ to rise a wee bit presents potential reconnection sites and does not add and twist or writhe to $C D$. Reconnecting at the right-hand site gives both reconnected tubes a -ve half turn (bottom configuration). It is interesting to note that if reconnection occurs at the left-hand site instead, both tubes would gain a positive half turn. The sense of all these twists would change if (in the middle panel) tube $C D$ was in front of tube $A B$.

\section{Discussion and Conclusions}

The expressions for self-helicity of reconnected flux tubes derived here should be a powerful tool for studying theoretical models of structures in the corona that undergo reconnection, e.g., Priest \& Longcope (2017). It is essential to accurately assess the amount of twist produced in such processes as this can have important consequences for the stability of flux tubes and their subsequent evolution.

Our results will also be important for interpreting simulation results and observations. Indeed, an example of 

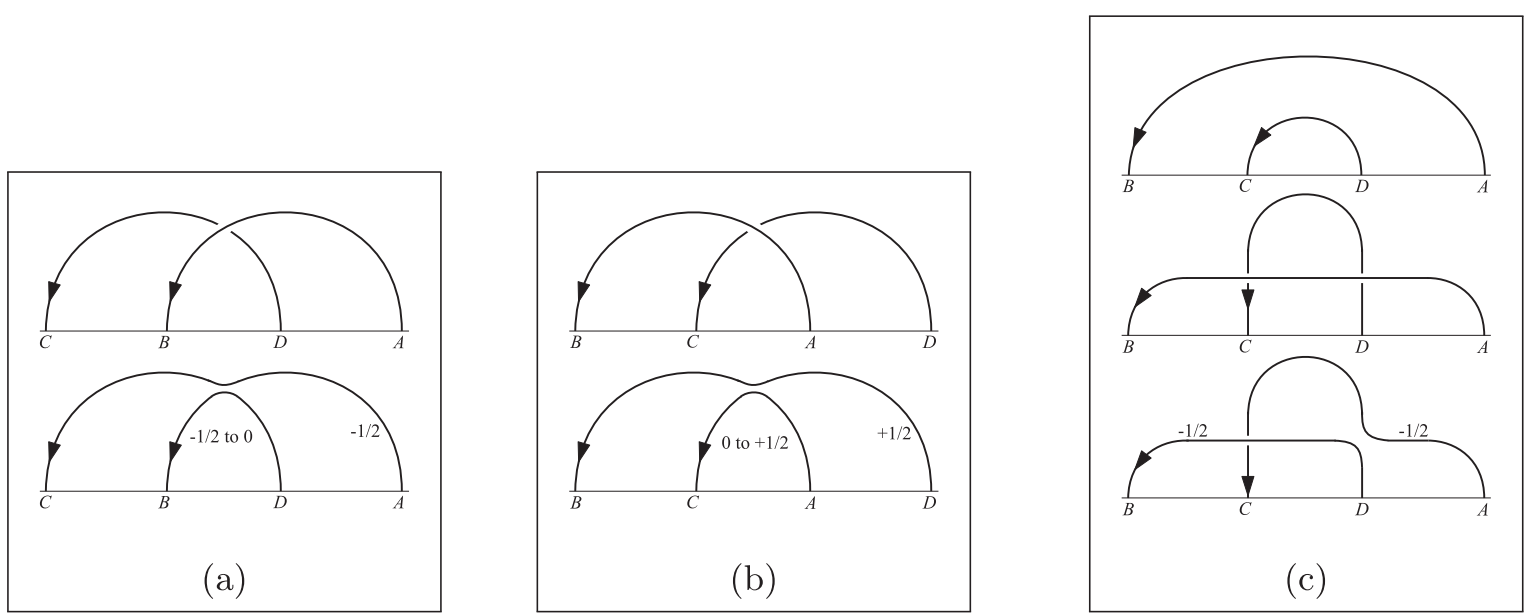

Figure 8. Three quasi-colinear footpoint configurations that guarantee a suitable reconnection site without the need for significant distortion of the flux tubes. (Only the tube axes are shown, and are viewed side-on.) The states before (top) and after (bottom) reconnection are shown. The signed value of the uniform twist equivalent to the self-helicity is also indicated.

nonequipartition of self-helicity in reconnected flux tubes was recently reported by Threlfall et al. (2018) who had a configuration resembling that in Figure 7 (a) but with $\theta_{A} \ll 1$, $\theta_{C} \ll 1, \theta_{B} \approx \theta_{D} \approx \pi / 2$, so similar to Figure 8(a). Our expectation (from (22)) is that $\tilde{H}_{A C} \approx-\Phi^{2} / 2$ and $\tilde{H}_{B D} \approx 0$. Threlfall et al. (2018) note that the underlying tube $(B D)$ in their simulation is untwisted, and their Figure 7 shows the field lines of the overlying tube having an obvious -ve twist of about the right amount. It should be noted that resistivity in their model may affect the twist in the reconnected tubes, and also that their simulation included footpoint motion, which may inject helicity, so a definitive comparison is not possible. However, the qualitative agreement with their results suggest the ideas in this paper may have relevance.

In conclusion, we have provided a theoretical analysis of the self-helicity and equivalent twist produced by reconnecting two flux tubes anchored to a plane such as the photosphere. If the tubes are initially crossed and untwisted, as in Figures 3(a) and 4(a),

1. The general criterion for equipartition of self-helicity between the reconnected flux tubes is that the footpoints form a suitable trapezium.

2. Nonequipartition of self-helicity is the usual situation, and can be extreme with either tube having no selfhelicity, but the other being twisted.

3. The amount of total self-helicity that can be deposited in the reconnected tubes ranges from zero to $2 \Phi^{2}$ dependent upon footpoint arrangement.

If the tubes are initially uncrossed but overlap, as in Figure 7,

1. Equipartition of self-helicity in the reconnected tubes is never achieved, although it may be approached in the limit of the footpoints having a particular quasi-colinear arrangement.

2. The overlying reconnected flux tube has a self-helicity whose magnitude always exceeds $\Phi^{2} / 2$ (i.e., a minimum of a half turn). The underlying tube's self-helicity magnitude never exceeds $\Phi^{2} / 2$, so always has less than a half turn.
If the tubes do not overlap, care should be taken to account for any twist or writhe that is introduced when bringing them together at the reconnection site.

A.N.W. was partially funded by STFC Consolidated Grant ST/N000609/1. The author thanks the referee for constructive comments and the suggestion to include Figure 5(c) and the associated discussion.

\section{ORCID iDs}

Andrew N. Wright (1) https://orcid.org/0000-0002-9877-1457

\section{References}

Berger, M. A. 1984, GApFD, 30, 79

Berger, M. A. 1986, GApFD, 34, 265

Berger, M. A. 1988, A\&A, 201, 355

Berger, M. A., \& Field, G. B. 1984, JFM, 147, 133

Calugareaunu, G. 1959, RRMPA, 4, 5

Demoulin, P., Pariat, E., \& Berger, M. A. 2006, SoPh, 233, 3

Finn, J. H., \& Antonsen, T. M. 1985, CoPPC, 9, 111

Hood, A. W., Browning, P. K., \& Van der Linden, R. A. M. 2009, A\&A, 506, 913

Jensen, T. H., \& Chu, M. S. 1984, PhFl, 27, 281

Moffatt, H. K. 1969, JFM, 34, 117

Moffatt, H. K., \& Ricca, R. L. 1995, RSPSA, 439, 411

Pariat, E., Demoulin, P., \& Berger, M. A. 2005, A\&A, 439, 1191

Priest, E. R., \& Longcope, D. W. 2017, SoPh, 292, 25

Priest, E. R., Longcope, D. W., \& Janvier, M. 2016, SoPh, 291, 2017

Russell, A. J. B., Yeates, A. R., Hornig, G., \& Wilmot-Smith, A. L. 2015, PhPl, 22, 032106

Song, Y., \& Lysak, R. L. 1989, JGR, 94, 5273

Taylor, J. B. 1974, PhRvL, 33, 1139

Threlfall, J., Hood, A. W., \& Priest, E. R. 2018, SoPh, 293, 98

White, J. H. 1969, AmJM, 91, 693

Wright, A. N. 1987, P\&SS, 35, 813

Wright, A. N. 1999, in Magnetic Helicity in Space and Laboratory Plasmas, ed. M. R. Brown, R. C. Canfield, \& A. A. Pevtsov (Washington, DC: AGU), 267

Wright, A. N., \& Berger, M. A. 1989, JGR, 94, 1295

Wright, A. N., \& Berger, M. A. 1990, JGR, 95, 8029

Yeates, A. R., \& Hornig, G. 2016, A\&A, 594, A98 